\begin{tabular}{|c|c|c|}
\hline CRITICAL & $\begin{array}{l}\text { ECOSYSTEM } \\
\text { PARTNERSHIP FUND }\end{array}$ & $\begin{array}{l}\text { Western Ghats } \\
\text { Special Series }\end{array}$ \\
\hline
\end{tabular}

\title{
Diversity AND distribution Of ANURANS IN PhANSAD WILDLIFE SANCTUARY (PWS), NORTHERN WESTERN GHATS OF INDIA
}

ISSN

Online 0974-7907

Print 0974-7893

OPEN ACCESS

\author{
Unmesh Katwate ${ }^{1}$, Deepak Apte ${ }^{2}$ \& Rupesh Raut ${ }^{3}$ \\ 1,2 Bombay Natural History Society (BNHS), Hornbill House, Opposite Lion Gate, Shaheed Bhagat Singh Road, Mumbai, \\ Maharashtra 400001, India \\ ${ }^{3}$ Department of Zoology, Elphinstone College, Mumbai, Maharashtra 400032, India \\ ${ }^{1}$ theunmesh@gmail.com (corresponding author), ${ }^{2}$ spiderconch@gmail.com, ${ }^{3}$ rupesh.raut@gmail.com
}

\begin{abstract}
In global consequences of rapidly changing climate and increased amphibian population decline, mapping amphibian diversity in biodiversity hotspots is essential. In this study we have systematically studied anurans of Phansad Wildlife Sanctuary in terms of species diversity, population structure, threat status and distribution. We recorded a total of 22 anuran species, of which 11 species are endemic to Western Ghats biodiversity hotspot. Family Dicroglossidae was found to be more species-rich. Spatial and temporal variation in anuran diversity was observed by using Shannon diversity and evenness indices. Most of the endemic and threatened anuran species are found to be associated with evergreen undisturbed forest patches. Habitat parameters like humidity, forest type, canopy coverage, riparian canopy coverage, stream persistence and litter depth are found to be major variables governing species diversity and distribution. Major anthropogenic threats to amphibians of Phansad Wildlife Sanctuary are discussed along with future conservation objectives. With range extension of species like Fejervarya caperata and Minervarya sahyadris further north in the Western Ghats, taxonomic ambiguities recorded during study are discussed briefly.
\end{abstract}

Keywords: Amphibians, diversity, endemism, species composition, threat status.

DOI: http://dx.doi.org/10.11609/JoTT.03038.3589-602 | ZooBank: urn:Isid:zoobank.org:pub:08F331D2-4CCB-4476-93FD-6CC7C934C75D

Editor: Sanjay Molur, ZOO/WILD, Coimbatore, India

Date of publication: 26 February 2013 (online \& print)

Manuscript details: Ms \# 03038 | Received 16 December 2011 | Final received 08 December 2012 | Finally accepted 31 December 2012

Citation: Katwate, U., D. Apte \& R. Raut (2013). Diversity and distribution of anurans in Phansad Wildlife Sanctuary (PWS), northern Western Ghats of India Journal of Threatened Taxa 5(2): 3589-3602; doi:10.11609/JoTT.03038.3589-602

Copyright: (C) Katwate et al. 2013. Creative Commons Attribution 3.0 Unported License. JoTT allows unrestricted use of this article in any medium, reproduction and distribution by providing adequate credit to the authors and the source of publication.

Funding: BNHS Conservation Department fund.

Competing Interest: None.

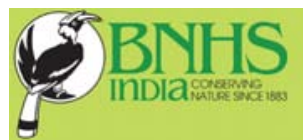

Author Contribution: UK and RR have contributed equally in extensive field studies, analysis of data and preparation of manuscript. DA gave important suggestions during field studies and preparation of manuscript.

Acknowledgements: We thank our co-researchers and field assistants from Bombay Natural History Society (BNHS) for their contribution in field surveys. We are grateful to our enthusiast wildlife photographer friends - Ajinkya Sawant, Saurabh Rane, Kunal Salunkhe, Aditya Sawant, Abhijit Gharat and Pritesh Nandvikar from Wild Explorers (WE) for their contribution in photo documentation of different amphibian species from study area. We are thankful to RFO and DFO, Maharashtra Forest Department for permit (Letter No. D-4/Land/1290, Dt. 15 October 2010) and field support; the museum and library authorities and the Director, Bombay Natural History Society for providing reference facilities and the constant encouragement, respectively; the Principal, Elphinstone College for necessary support; Neelesh Dahanukar for providing valuable guidelines; and the anonymous reviewers for constructive comments on the manuscript.

Author Details: UNMESH KATWATE is a zoology postgraduate currently working as Scientist-A in BNHS. He is studying ecology and conservation aspects of freshwater fishes and amphibians in northern Western Ghats and currently working on diversity and ecology of costal plateaus of Konkan in Western Ghats. DEEPAK APTE a marine biologist and conservationist, currently a Chief Operating Officer (COO) of BNHS.

RUPESH RAUT is a head of Department of Zoology, Elphinstone College. He works on ecology, systematic biology and molecular phylogeny of freshwater fishes and amphibians of Western Ghats.

The publication of this article is supported by the Critical Ecosystem Partnership Fund (CEPF), a joint initiative of l'Agence Française de Développement, Conservation International, the European Commission, the Global Environment Facility, the Government of Japan, the MacArthur Foundation and the World Bank. 


\section{INTRODUCTION}

The Western Ghats of India, ranked among 34 biodiversity hotspots (Mittermeier et al. 2005), are well known for rich amphibian diversity. Around 6,771 amphibian species are listed throughout the world, of which 333 species are recorded from India (Frost 2011). With the addition of one Raorchestes (Seshadri et al. 2012) the total number of amphibian species listed in the Western Ghats are 183, among them 162 (88.5\%) are endemic (Gururaja 2012). Deforestation, changes in land use, urbanization and industrialization are major threats for the amphibians of Western Ghats (Daniels 1991; Ghate \& Padhye 1996). Recent record of pathogenic fungus is also alarming (Nair et al. 2011). Out of total $160,000 \mathrm{~km}^{2}$ area of the Western Ghats only 9\% area is protected under national parks and wildlife sanctuaries (Gunawardene et al. 2007) therefore management of biological resources is critical in the Western Ghats. To implement strong policies for amphibian conservation in the Western Ghats, systematic study, spatial mapping and long term monitoring of amphibian species are needed (Padhye \& Ghate 2002; Dahanukar \& Padhye 2005).

Amphibians of northern Western Ghats are relatively less studied as compared to the central and the southern Western Ghats. Species discoveries like Gegeneophis nadkarnii (Bhatta \& Prashanth, 2004), Indotyphlus maharashtraensis (Giri et al., 2004), Gegeneophis goaensis (Bhatta et al., 2007), Pseudophilautus amboli (Biju \& Bossuyt, 2009) and Nyctibatrachus danieli (Biju et al., 2011) are significant findings from this region. Taxonomic as well as certain ecological studies by Daniel (1974), Yazdani \& Mahabal (1976), Ravichandran \& Pillai (1990), Daniels (1992), Ghate \& Padhye (1996), Sekar (1999), Padhye et al. (2002), Dahanukar \& Padhye (2005), Biju \& Bossuyt (2009), Kumbar \& Patil (2010) and Biju et al. (2011) in Maharashtra region of northern Western Ghats are mostly confined to Bhimashankar, Mulshi region of Pune District, Satara, Sangli District and Amboli region of Sindhudurg District. Knowledge of amphibian diversity and its species assemblage structure is still unknown from other parts of northern Western Ghats, which is especially true for the Konkan region.

Gokhale \& Velankar (1996) demarcated Phansad Wildlife Sanctuary (PWS) as a highly diverse region in Western Ghats because of its unique evergreen type of vegetation. Presence of regionally endangered and endemic semi-evergreen to evergreen plant communities rank this area to be one among the highest conservation priority zones of Maharashtra (Rodgers \& Panwar 1998).
Although forests of PWS are not directly connected to the main Western Ghats range but the similarity in the occurrence of flora and fauna is striking (Rodgers \& Panwar 1988; Pande \& Pathak 2005). Diversity and distribution of amphibians in PWS is not fully understood yet. In this study we have systematically documented diversity and distribution pattern of anurans in PWS. To our knowledge this is the first systematic effort ever taken to document amphibian fauna in PWS.

\section{MATERIALS AND METHODS}

\section{Study area}

PWS is situated on the lower hills of the Konkan coast, west of Sahyadri and it covers about $52.66 \mathrm{~km}^{2}$ area in the Raigad District, Maharashtra. It lies between $18.33-18.5^{\circ} \mathrm{N} \& 72.90-73.0625^{\circ} \mathrm{E}$ with altitude ranging from $20 \mathrm{~m}$ to $300 \mathrm{~m}$. Annual rainfall ranges between $2162 \mathrm{~mm}$ and $3469 \mathrm{~mm}$. Parts of the study area have perennial sources of water bearing old evergreen type of forest. Small hilly regions and slopes show Memecylon umbellatum tree dominating the stunted evergreen type of forest. Plateau regions shows grassland type of habitat whereas boundary line areas of PWS are dominated by degraded moist deciduous type of forest. We have selected a total of eight different localities in the sanctuary area representing different habitats (Table 1 \& Fig. 1). We surveyed this area from June to September 2011. Even though our study period was limited to four months, significant efforts were taken to give a broad exposure on status of amphibian fauna of PWS.

\section{Data collection and analysis}

Anuran species diversity in PWS was studied using two different methods. Ad-hoc search method was used to prepare a checklist of species present in the study area and belt transect method was used to quantify anuran diversity in different locations in PWS. Surveys were conducted mostly during late evening and early night; some areas were also searched during day time. At all sampling locations different habitat features like floor litter, underside rocks and boulders, tree barks and leaves, water pools, rocky crevices near flowing streams were documented and scanned for anurans. Sampling was performed by hand picking the specimen. Efforts were maintained to identify specimens up to species level in the field, body measurements of species taken with a digital caliper and individuals were released back at original location. In case of unidentified species representative voucher specimens were collected, 
fixed in $4 \%$ formalin and stored in $70 \%$ alcohol. IUCN guidelines were followed for scientific collection of some threatened anuran species (IUCN 2011). Total of 3260 individuals were recorded of which only 17 were preserved for further investigation and others were released in the same location. Voucher specimens were deposited at Konkan field station museum of Bombay Natural History Society, Ratnagiri (accession numbers BNHS A1 to BNHS A17). Identification of specimens was done using standard taxonomic literatures like Boulenger (1890), Annandale (1919), Daniel (1963 a,b; 1975), Daniel \& Sekar (1989), Bossuyt \& Dubois (2001), Biju \& Bossuyt (2009), and Biju et al. (2011). The threat status of the anuran species is adopted from IUCN Red List of Threatened Species-Version 2011.1 (IUCN 2011). Site specific anthropogenic threats observed in the study sites are listed in Table 1.

Biological parameters like percentage canopy cover, forest type, forest floor litter depth, riparian canopy cover (10m on both sides of the main stream) and physical parameters like altitude, atmospheric temperature and relative percentage humidity were recorded for each species during the study. Temperature and relative percentage humidity were recorded using digital hygro-thermometer. Canopy cover was estimated using spherical densiometer. All habitat variables were graded from lowest to highest values. Details of habitat guild classification are depicted in Table 2. Canonical Correspondence Analysis (CCA) has been proven to be one of best multivariate statistical tool to extract correlation between biological assemblage of species and their environment (Gururaja \& Ramachandra 2012). We analyzed the relationship between biological and physical habitat parameters, study sites and species abundance data through CCA by using PAST ver1.98 (Hammer et al. 2001). Quantification of anuran diversity was performed by using the belt transect method. During the study period, two 100 $5 \mathrm{~m}$ belt transects were laid at each study location every month. Transects were laid in such a way that they would overlap all possible breeding habitats. Active search for amphibians on transect were conducted by four people from 1700-2300 hr. Number of species and abundance of each species were recorded for each transect by visual encounter. Actual number

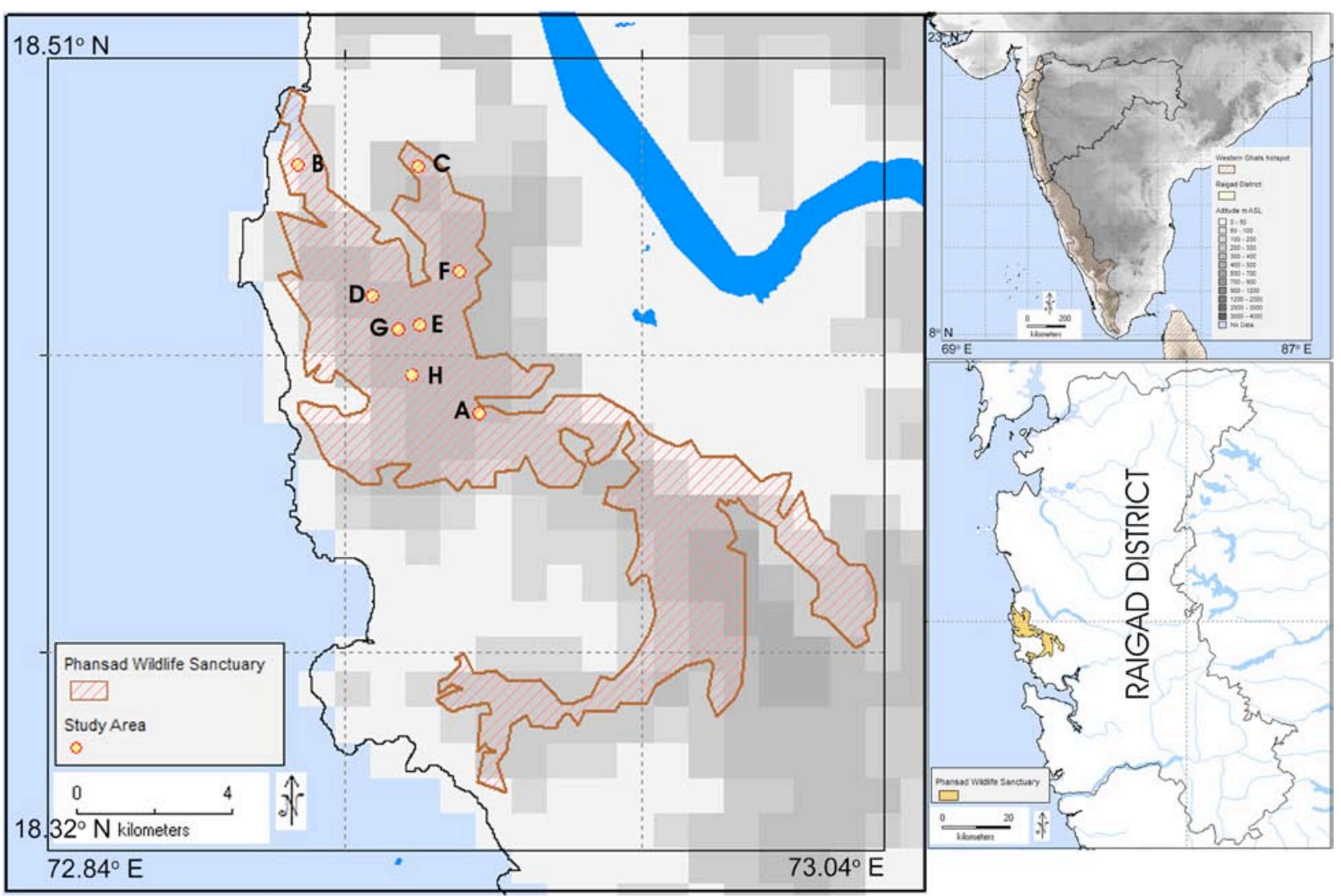

Figure 1. Sampling locations in Phansad Wildlife Sanctuary

A - Supegaon, B - Barshiv, C - Par Gaan, D - Chikhal Gaan, E - Phansad Gaan, F - Savarat Gaan, G - Chakacha Maal, H - Ghunyacha Maal. 
Table 1. Habitat parameters recorded at the study sites.

\begin{tabular}{|c|c|c|c|c|c|c|c|c|}
\hline \multirow{2}{*}{$\begin{array}{l}\text { Habitat } \\
\text { parameters }\end{array}$} & \multicolumn{8}{|c|}{ Study sites } \\
\hline & Supegaon & Barshiv & Par Gaan & Chikhal Gaan & $\begin{array}{c}\text { Phansad } \\
\text { Gaan }\end{array}$ & Savarat Gaan & $\begin{array}{c}\text { Chakacha } \\
\text { Maal }\end{array}$ & $\begin{array}{c}\text { Ghunyacha } \\
\text { Maal }\end{array}$ \\
\hline Lat./long. & $\begin{array}{l}18.42^{\circ} \mathrm{N} \\
72.95^{\circ} \mathrm{E}\end{array}$ & $\begin{array}{l}18.48^{\circ} \mathrm{N} \\
72.90^{\circ} \mathrm{E}\end{array}$ & $\begin{array}{l}18.48^{\circ} \mathrm{N} \\
72.93^{\circ} \mathrm{E}\end{array}$ & $\begin{array}{l}18.45^{\circ} \mathrm{N} \\
72.92^{\circ} \mathrm{E}\end{array}$ & $\begin{array}{l}18.44^{\circ} \mathrm{N} \\
72.93^{\circ} \mathrm{E}\end{array}$ & $\begin{array}{l}18.46^{\circ} \mathrm{N} \\
72.94^{\circ} \mathrm{E}\end{array}$ & $\begin{array}{l}18.44^{\circ} \mathrm{N} \\
18.44^{\circ} \mathrm{E}\end{array}$ & $\begin{array}{l}18.43^{\circ} \mathrm{N} \\
72.93^{\circ} \mathrm{E}\end{array}$ \\
\hline $\begin{array}{l}\text { Canopy coverage } \\
\text { (\%) }\end{array}$ & $40-60$ & $20-50$ & $40-60$ & 80-91 & $70-80$ & $60-80$ & Nil & Nil \\
\hline $\begin{array}{l}\text { Riparian canopy } \\
\text { coverage (\%) }\end{array}$ & $50-63$ & $0-40$ & $30-63$ & $83-91$ & $78-87$ & $75-87$ & Nil & Nil \\
\hline $\begin{array}{l}\text { Forest floor litter } \\
\text { depth }(\mathrm{mm})\end{array}$ & $0-100$ & $0-50$ & $0-100$ & $100-200$ & 70-150 & $70-180$ & Nil & Nil \\
\hline $\begin{array}{l}\text { Altitude } \\
\text { (m) }\end{array}$ & 245 & 104 & 159 & 236 & 237 & 227 & 281 & 294 \\
\hline $\begin{array}{l}\text { Atmospheric } \\
\text { temperature }\left({ }^{\circ} \mathrm{C}\right)\end{array}$ & $29-32$ & $30-33$ & $28-31$ & $26-29$ & $27-29$ & $27-29$ & $28-33$ & $28-33$ \\
\hline Humidity (\%) & $70-86$ & $68-83$ & $77-85$ & $88-97$ & $89-97$ & $87-97$ & $75-90$ & $75-91$ \\
\hline Stream type & $\begin{array}{l}\text { secondary } \\
\text { streams }\end{array}$ & $\begin{array}{l}\text { ephemeral } \\
\text { primary } \\
\text { stream }\end{array}$ & $\begin{array}{l}\text { ephemeral } \\
\text { primary } \\
\text { stream }\end{array}$ & $\begin{array}{l}\text { perennial } \\
\text { primary } \\
\text { stream }\end{array}$ & $\begin{array}{l}\text { perennial } \\
\text { primary } \\
\text { stream }\end{array}$ & $\begin{array}{l}\text { perennial } \\
\text { primary } \\
\text { stream }\end{array}$ & Nil & Nil \\
\hline Stream habitat & cascade & cascade & $\begin{array}{l}\text { cascade, run } \\
\text { and dammed } \\
\text { pools }\end{array}$ & $\begin{array}{l}\text { cascade, run } \\
\text { and dammed } \\
\text { pools }\end{array}$ & $\begin{array}{c}\text { cascade, run } \\
\text { and dammed } \\
\text { pools }\end{array}$ & $\begin{array}{l}\text { cascade, run } \\
\text { and dammed } \\
\text { pools }\end{array}$ & Nil & Nil \\
\hline Forest type & $\begin{array}{l}\text { degraded } \\
\text { moist } \\
\text { deciduous } \\
\text { forest, semi- } \\
\text { evergreen } \\
\text { forest }\end{array}$ & $\begin{array}{l}\text { degraded } \\
\text { moist } \\
\text { deciduous } \\
\text { forest }\end{array}$ & $\begin{array}{l}\text { degraded } \\
\text { moist } \\
\text { deciduous } \\
\text { forest }\end{array}$ & $\begin{array}{l}\text { evergreen } \\
\text { forest }\end{array}$ & $\begin{array}{l}\text { evergreen } \\
\text { forest }\end{array}$ & $\begin{array}{l}\text { evergreen } \\
\text { forest }\end{array}$ & grassland & grassland \\
\hline $\begin{array}{l}\text { Observed } \\
\text { disturbances }\end{array}$ & $\begin{array}{l}\text { deforestation } \\
\text { for fire wood } \\
\text { purpose, } \\
\text { cattle grazing } \\
\text { in forest area, } \\
\text { laterite soil } \\
\text { and boulder } \\
\text { mining }\end{array}$ & $\begin{array}{l}\text { deforestation } \\
\text { by slash and } \\
\text { burn, rice } \\
\text { cultivation } \\
\text { in reserved } \\
\text { forest area, } \\
\text { cattle grazing }\end{array}$ & $\begin{array}{l}\text { deforestation } \\
\text { by slash } \\
\text { and burn } \\
\text { method, rice } \\
\text { farming, shift } \\
\text { cultivation }\end{array}$ & Nil & Nil & $\begin{array}{c}\text { cattle grazing, } \\
\text { hunting are } \\
\text { predominant } \\
\text { human } \\
\text { disturbances, } \\
\text { deforestation } \\
\text { for fire wood } \\
\text { purpose }\end{array}$ & cattle grazing & cattle grazing \\
\hline
\end{tabular}

of individuals encountered during transect sampling were used to calculate percent species abundance at each study location in every month of the study period. Quantitative data generated from transect studies were used to calculate diversity and evenness indices (Magurran 1988). The Shannon index of diversity was calculated using the equation:

$$
H^{\prime}=-\sum p_{i}\left(\ln p_{i}\right) \text {, }
$$

where $p_{i}=n_{i} / N$ and $n_{i}$ is the number of individuals of ith species and $N=\sum n_{i}$. Evenness index was calculated by the equation:

$$
E=H^{\prime} / \ln S \text {. }
$$

Cluster plot indicating species composition similarity between different study locations was generated using Bray-Curtis similarity index (Magurran 1988). Observed species abundance may not always represent the true abundance of the communities therefore adequacy of sampling efforts was assessed by using species accumulation curves. Here, we have used EstimateS (version 8.0.0) software developed by Colwell (2006) for generating species accumulation curves, which uses Monte Carlo simulations of random samples drawn from the total set of samples for estimating the average species richness. We ran 200 randomizations for a given number of samples to estimate the species richness values and their means which were used for plotting species accumulation curves with respect to number of samples as well as against number of individuals recorded during study period. Species accumulation curves were found to be best fitted by using MichaelisMenton equation

$$
\mathrm{S}_{\text {obs }}=\mathrm{S}_{\max } \mathrm{n} /(\mathrm{B}+\mathrm{n}) \text {, }
$$

where $S_{\max }$ is the maximum number of species, $n$ is the number of individuals in the set of samples so far, 
Table 2. Biological and physical habitat variables guild gradation for anurans recorded in study area.

\begin{tabular}{|c|c|c|}
\hline \multicolumn{3}{|c|}{ Biological habitat variables } \\
\hline Canopy coverage (\%) & $<50$ & 1 \\
\hline & $50-80$ & 2 \\
\hline & 80-100 & 3 \\
\hline \multirow[t]{3}{*}{ Riparian canopy coverage (\%) } & $<50$ & 1 \\
\hline & $50-80$ & 2 \\
\hline & $80-100$ & 3 \\
\hline \multirow[t]{3}{*}{ Forest type } & Grassland/plateau & 1 \\
\hline & Moist/semi-deciduous & 2 \\
\hline & Evergreen & 3 \\
\hline \multirow[t]{3}{*}{ Forest floor litter (mm) } & $<50$ & 1 \\
\hline & $50-100$ & 2 \\
\hline & $100-200$ & 3 \\
\hline \multirow[t]{2}{*}{ Stream persistence } & Seasonal & 1 \\
\hline & Perennial & 2 \\
\hline \multicolumn{3}{|l|}{ Physical habitat variables } \\
\hline \multirow[t]{3}{*}{ Altitude (m) } & $<100$ & 1 \\
\hline & $100-200$ & 2 \\
\hline & $200-300$ & 3 \\
\hline \multirow[t]{3}{*}{ Relative humidity (\%) } & $<60$ & 1 \\
\hline & $60-80$ & 2 \\
\hline & $80-100$ & 3 \\
\hline
\end{tabular}

and $B$ is the number of individuals needed to get half the maximum number of species, ie. When $n=B$, Sobs $=\mathrm{S}_{\max } / 2$. Geographical map of study site (Fig. 1) was prepared by using DIVA-GIS (Hijmans et al. 2002).

\section{RESULTS AND DISCUSSION}

In the four-month study period we documented a total of 22 anuran species belonging to seven families and 15 genera (Table 3, Images 1-12). Family Dicroglossidae was found to be more species-rich covering about $41 \%$ (nine species) of the total anuran species recorded. Family Rhacophoridae was the second most speciesrich family in our study area covering $23 \%$ of total anuran species (five species of arboreal frog). Family Microhylidae and Ranixalidae contributed $13.63 \%$ and $9.09 \%$, respectively to the total anuran species found in the study area. Bufonidae, Nyctibatrachidae and Ranidae contributed $4.5 \%$ individually to total anuran species. We have reported total 11 anuran species (50\% of total) from PWS showing exclusive endemism to Western Ghats. In the transect study we documented total 22 anuran species with an abundance of 3260 individuals in 64 samples. Our study period was restricted to four months only therefore there are possibilities of missing some rare and cryptic species during sampling efforts. Figs. 2a \& $2 b$ shows species accumulation curves plotted against number of samples (a) and number of individuals (b). The curves were found to best fit $\left(R^{2}=0.89\right)$ with $S_{\text {max }}$ value at 22 .

Study site $A$ and $F$ reported 15 and 14 species respectively. Minimum species were observed at sites $G$ and $H$ with 10 species each. Shannon $H^{\prime}$ diversity index values also predict the same pattern. Site $A$ has highest $H^{\prime}$ value (1.01), while site $G$ and $H$ have lowest index values with 0.86 and 0.87. Despite of low species richness sites $C, D$ and $E$ have more evenly distributed species abundance with an $E$ index value $(S=11, E=0.94)$, $(\mathrm{S}=11, \mathrm{E}=0.93$ ) and $(\mathrm{S}=11, \mathrm{E}=0.89$ ) (Table 4). Anurans at site $D$ and $E$ show maximum species endemism with seven species followed by site $F$ and $A$ having six endemics of Western Ghats. Table 5 reveals that species richness and $\mathrm{H}^{\prime}$ index value are found to be almost equal

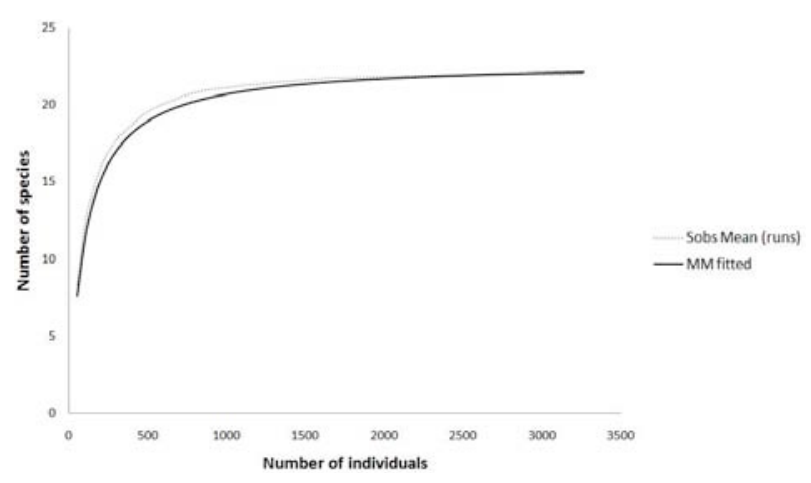

Figure $\mathbf{2}$ (a): Species accumulation curve - individuals vs. species richness (dotted grey line indicates observed species mean and black solid line indicates Michaelis-Menten mean).

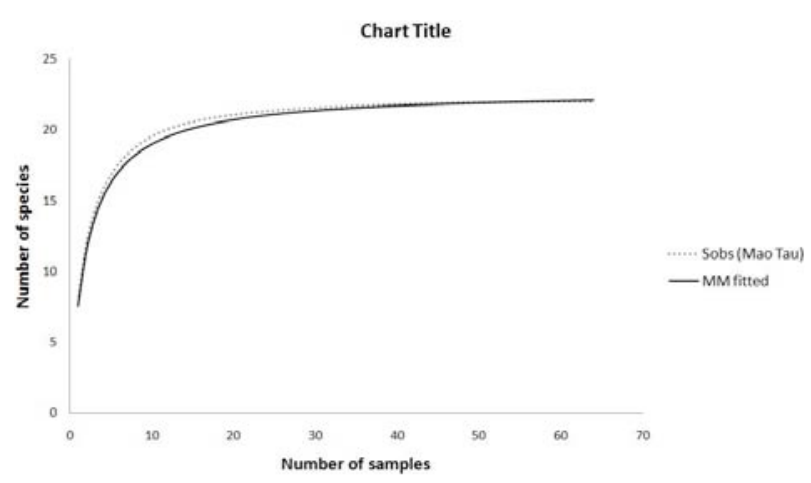

Figure 2(b). Species accumulation curve - samples vs. species richness (dotted grey line indicates observed species mean and black solid line indicates Michaelis-Menten mean). 
Table 3. Anuran abundance recorded at different study localities in Phansad Wildlife Sanctuary (PWS).

\begin{tabular}{|c|c|c|c|c|c|c|c|c|c|}
\hline \multirow{2}{*}{ Family/species $^{\text {a }}$} & \multicolumn{8}{|c|}{ Anuran abundance at different study localities ${ }^{b}$} & \multirow{2}{*}{$\begin{array}{l}\text { Global } \\
\text { Status }^{c}\end{array}$} \\
\hline & A & B & C & D & E & $\mathbf{F}$ & G & H & \\
\hline \multicolumn{10}{|l|}{ Bufonidae } \\
\hline Duttaphrynus melanostictus (Schneider, 1799) & 40 & 25 & 16 & 0 & 0 & 9 & 0 & 0 & LC \\
\hline \multicolumn{10}{|l|}{ Dicroglossidae } \\
\hline Euphlyctis cyanophlyctis (Schneider, 1799) & 113 & 150 & 48 & 0 & 0 & 55 & 94 & 100 & LC \\
\hline Fejervarya cf. keralensis* (Dubois, 1980) & 32 & 2 & 25 & 0 & 0 & 0 & 26 & 26 & LC \\
\hline $\begin{array}{l}\text { Fejervarya caperata* (Kuramoto, Joshy, Kurabayashi \& } \\
\text { Sumida, 2007) }\end{array}$ & 4 & 0 & 0 & 0 & 0 & 0 & 26 & 24 & DD \\
\hline Fejervarya rufescens* (Jerdon, 1853) (Image 1) & 56 & 47 & 33 & 51 & 29 & 7 & 86 & 66 & LC \\
\hline Fejervarya syhadrensis (Annandale, 1919) & 82 & 43 & 40 & 0 & 0 & 6 & 84 & 52 & LC \\
\hline Hoplobatrachus tigerinus (Daudin, 1803) & 30 & 54 & 19 & 0 & 0 & 29 & 16 & 12 & LC \\
\hline Minervarya sahyadris* (Dubois et al. 2001) (Image 2) & 83 & 41 & 90 & 0 & 0 & 27 & 102 & 80 & EN \\
\hline Sphaerotheca breviceps (Schneider, 1799) (Image 3) & 83 & 45 & 23 & 0 & 0 & 10 & 46 & 39 & LC \\
\hline Sphaerotheca dobsonii (Boulenger, 1882) (Image 4) & 6 & 0 & 0 & 40 & 25 & 0 & 2 & 1 & LC \\
\hline \multicolumn{10}{|l|}{ Microhylidae } \\
\hline Microhyla ornata (Dumeril \& Bibron, 1841) (Image 5) & 0 & 0 & 0 & 0 & 0 & 0 & 11 & 17 & LC \\
\hline Ramanella mormorata* (Jerdon, 1854) (Image 6) & 4 & 1 & 0 & 0 & 0 & 0 & 0 & 0 & EN \\
\hline Uperodon globulosus (Gunther, 1864) (Image 7) & 3 & 0 & 0 & 0 & 0 & 0 & 0 & 0 & LC \\
\hline \multicolumn{10}{|l|}{ Nyctibatrachidae } \\
\hline $\begin{array}{l}\text { Nyctibatrachus humayuni* (Bhaduri \& Kripalani, 1955) } \\
\text { (Image 8) }\end{array}$ & 0 & 0 & 0 & 18 & 7 & 0 & 0 & 0 & VU \\
\hline \multicolumn{10}{|l|}{ Ranidae } \\
\hline Hylarana malabarica* (Tschudi, 1838) & 14 & 14 & 13 & 24 & 13 & 3 & 0 & 0 & LC \\
\hline \multicolumn{10}{|l|}{ Ranixalidae } \\
\hline Indirana beddomii* (Gunther, 1875) (Image 9) & 0 & 0 & 0 & 30 & 23 & 3 & 0 & 0 & LC \\
\hline Indirana leithii* (Boulenger, 1888) & 0 & 0 & 0 & 16 & 10 & 1 & 0 & 0 & VU \\
\hline \multicolumn{10}{|l|}{ Rhacophoridae } \\
\hline Polypedates maculatus (Gray, 1834) & 48 & 46 & 14 & 45 & 66 & 33 & 0 & 0 & LC \\
\hline Polypedates cf. maculatus (Gray, 1834) & 0 & 0 & 0 & 15 & 8 & 1 & 0 & 0 & LC \\
\hline Pseudophilautus cf. amboli* (Biju \& Bossuyt, 2009) (Image 10) & 0 & 0 & 0 & 38 & 32 & 2 & 0 & 0 & $\mathrm{CR}$ \\
\hline Raorchestes bombayensis* (Annandale, 1919) (Image 11) & 17 & 0 & 8 & 98 & 68 & 45 & 0 & 0 & VU \\
\hline Raorchestes cf. bombayensis* (Annandale, 1919) (Image 12) & 0 & 0 & 0 & 28 & 23 & 0 & 0 & 0 & Vu \\
\hline
\end{tabular}

* Species endemic to Western Ghats.

a - Taxonomic status adapted from Boulenger (1890), Annandale (1919), Daniel (1963 a,b; 1975), Daniel \& Sekar (1989), Bossuyt \& Dubois (2001), Biju \& Bossuyt (2009), and Biju et al. (2011).

b - Study area: A - Supegaon; B - Barshiv; C - Par Gaan; D - Chikhal Gaan; E - Phansad Gaan; F - Savarat Gaan; G - Chakacha Maal; H - Ghunyacha Maal. c - Global status adapted IUCN 2011. IUCN Red List of Threatened Species. Version 2011.1. <www.iucnredlist.org>. Downloaded on 03 October 2011. LC Least Concern, DD- Data Deficient, VU- Vulnerable, EN- Endangered, CR- Critically Endangered.

throughout June, July and August with $\left(\mathrm{S}=21, \mathrm{H}^{\prime}=1.14\right)$, $\left(\mathrm{S}=20, \mathrm{H}^{\prime}=1.15\right)$ and $\left(\mathrm{S}=21, \mathrm{H}^{\prime}=1.11\right)$, respectively. $\mathrm{A}$ drastic fall in species richness and $H^{\prime}$ index value was observed in the month of September $\left(S=15, H^{\prime}=0.92\right)$. Similar pattern was found to be followed by evenness in anuran abundance with $\mathrm{E}=0.78$.

Our study period covers wet period of northern Western Ghats as it starts from June as south-west monsoon and ends in September. It has been already known that frogs in northern Western Ghats reproduce mainly in June and July because of longer dry period (Dahanukar \& Padhye 2005). Maximum abundance was found in the month of June, about 1167 individuals were recorded. It is possibly because June marks the onset of south-west monsoon and it's a peak time for amphibian reproduction. Furthermore no drastic fluctuations in 


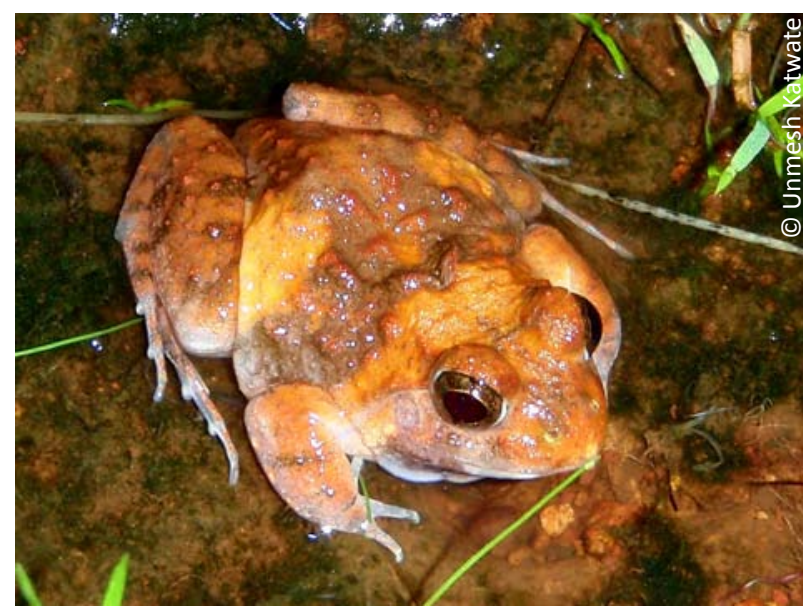

Image 1. Fejervarya rufescens

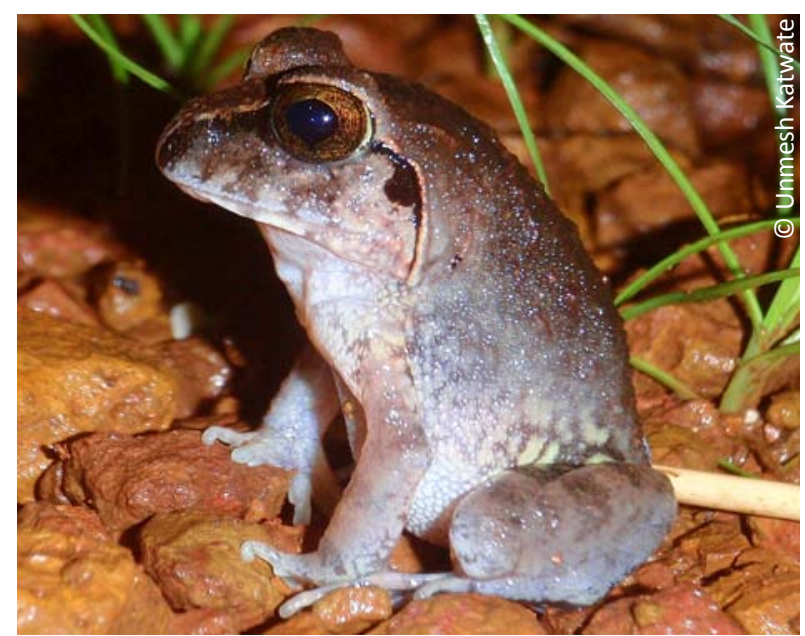

Image 3. Sphaerotheca breviceps

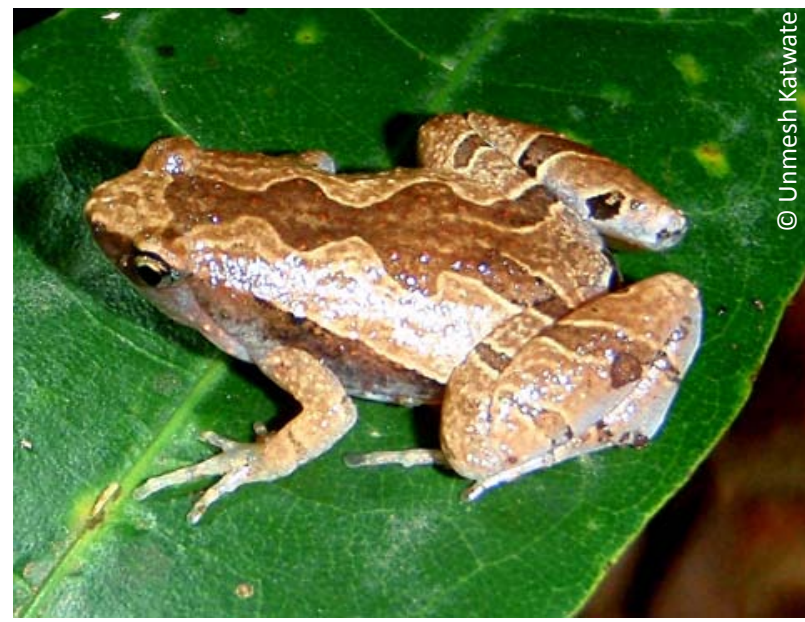

Image 5. Microhyla ornata

abundance were observed from July to September but species diversity in the month of September declines

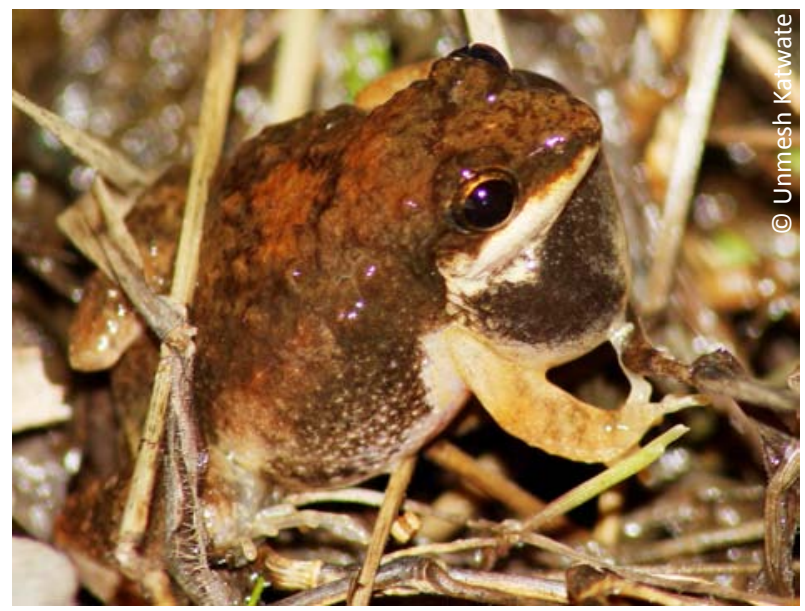

Image 2. Minervarya sahyadris

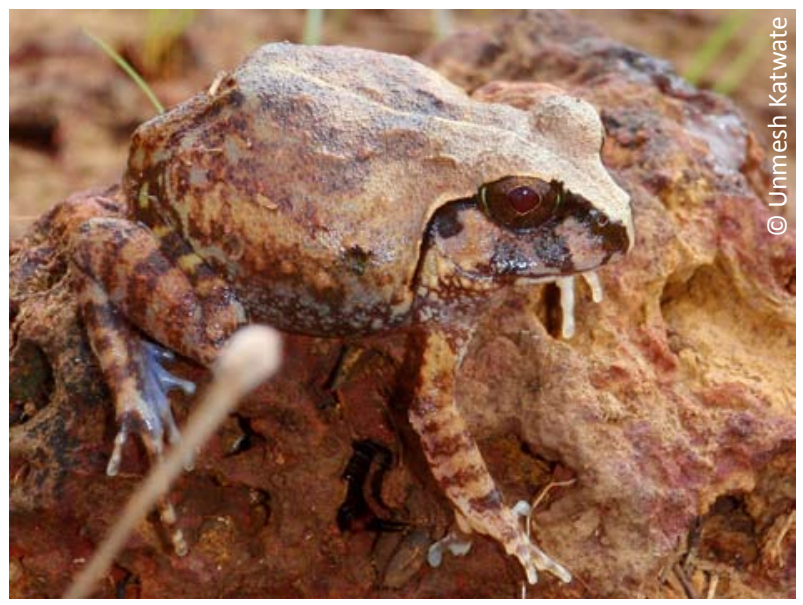

Image 4. Sphaerotheca dobsonii

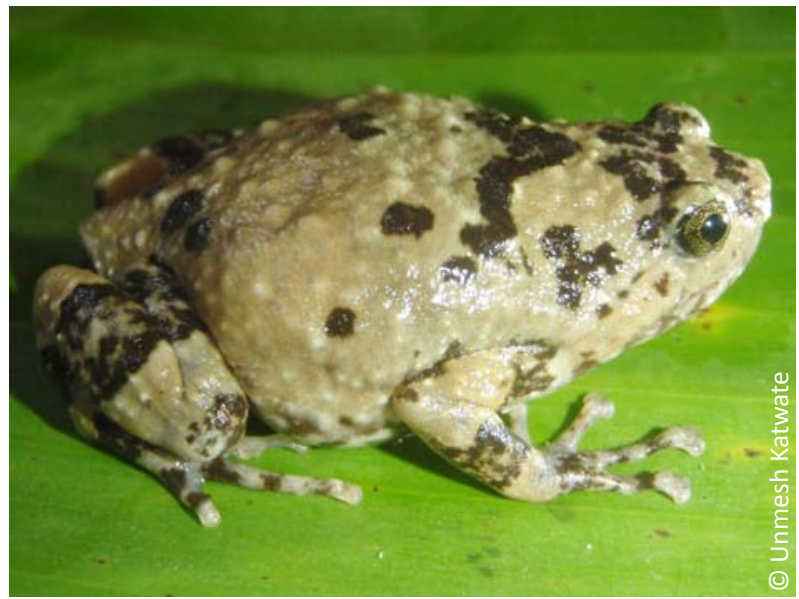

Image 6. Ramanella mormorata

drastically, only 15 species were observed in this month (Table 5). Fall in species richness, Shannon diversity and 

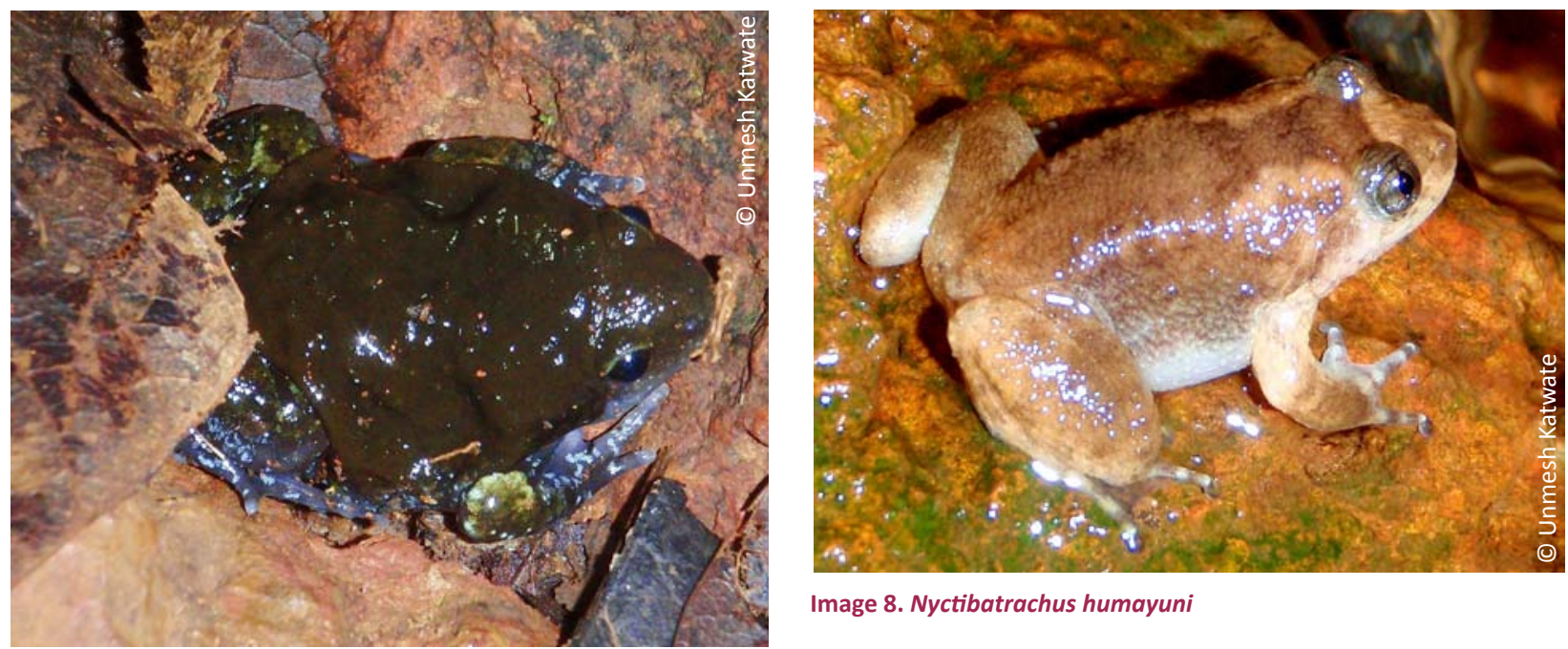

Image 8. Nyctibatrachus humayuni

Image 7. Uperodon globulosus
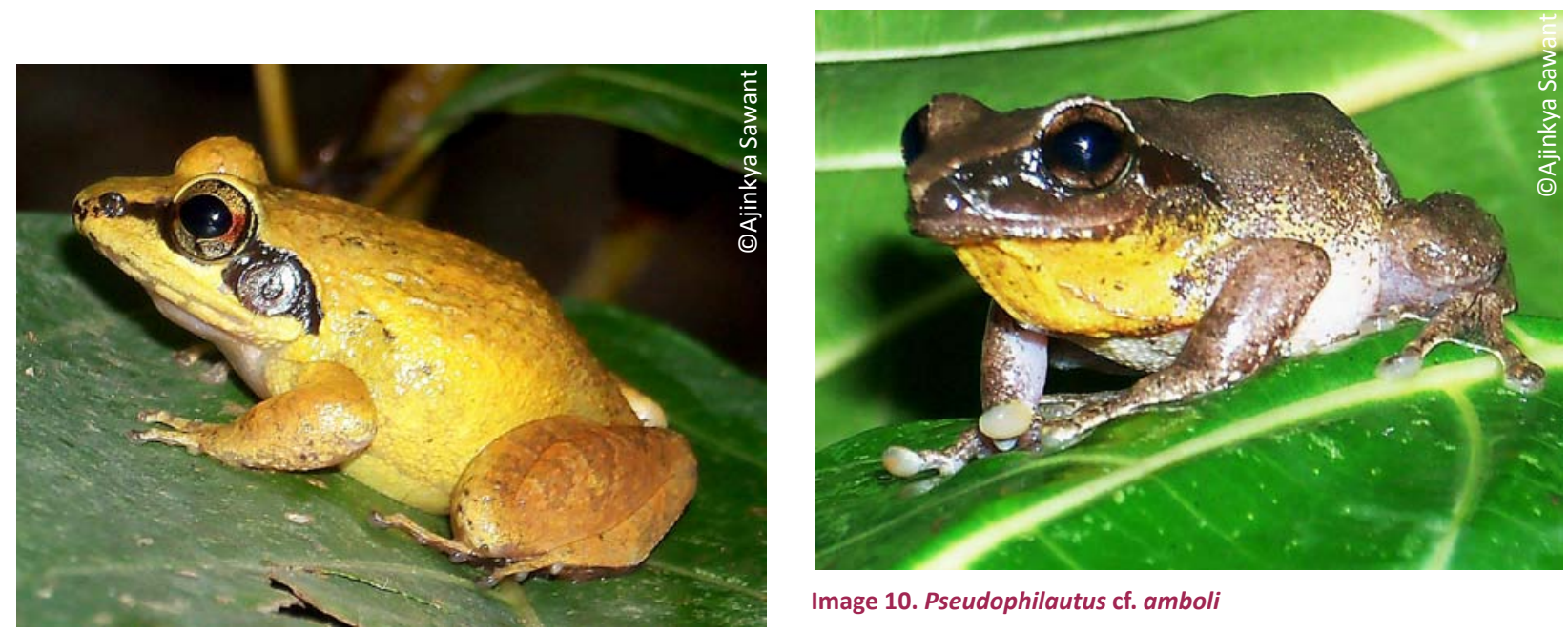

Image 10. Pseudophilautus cf. amboli
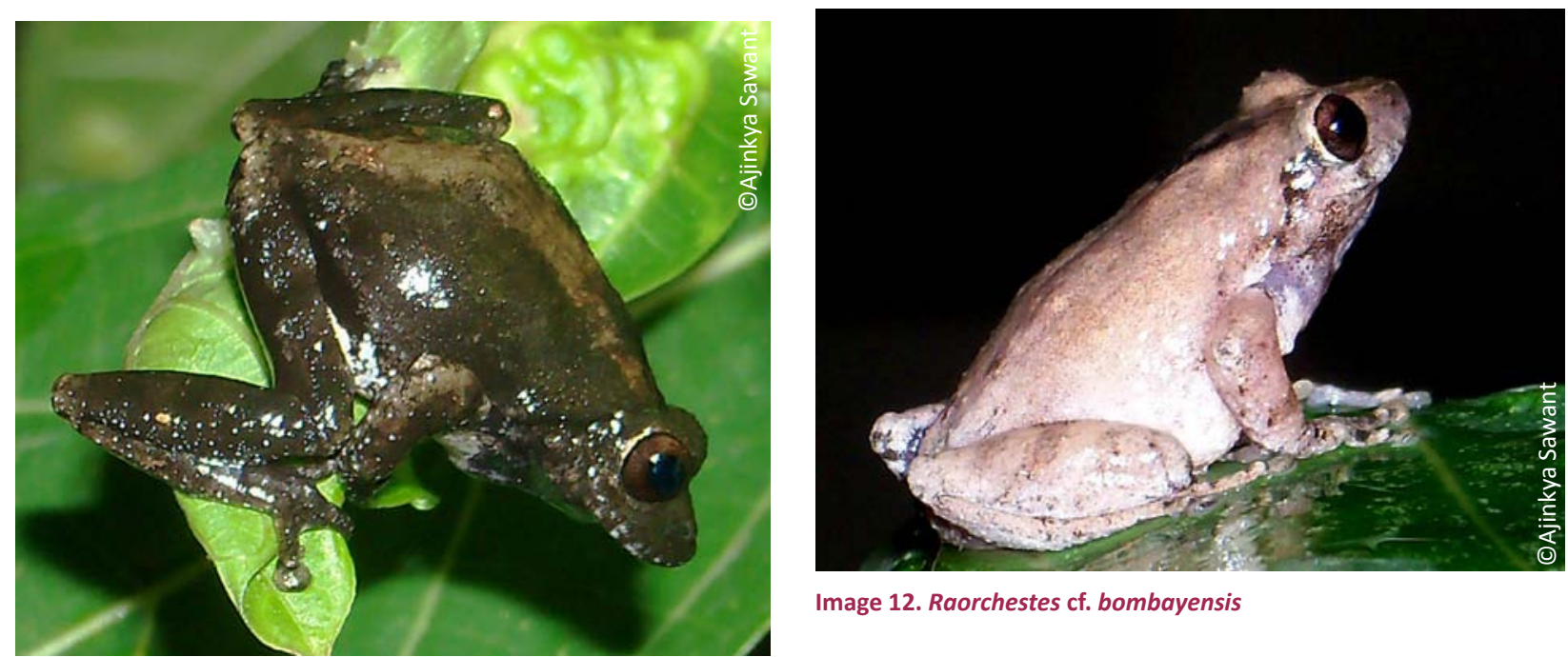

Image 12. Raorchestes cf. bombayensis

Image 11. Raorchestes bombayensis 
Table 4. Variation in species abundance, endemism, Shannon index and evenness index at various study locations in PWS.

\begin{tabular}{|l|c|c|c|c|c|c|c|c|}
\hline Study sites & A & B & C & D & E & F & G & H \\
\hline Species & 15 & 11 & 11 & 11 & 11 & 14 & 10 & 10 \\
\hline Individuals & 615 & 468 & 329 & 403 & 304 & 231 & 493 & 417 \\
\hline Endemic species & 6 & 4 & 4 & 7 & 7 & 6 & 4 & 4 \\
\hline Endemic species (\%) & 40 & 36.3 & 36.3 & 63.6 & 63.6 & 42.8 & 40 & 40 \\
\hline Shannon H' index & 1.016 & 0.881 & 0.942 & 0.972 & 0.935 & 0.918 & 0.868 & 0.876 \\
\hline Evenness index & 0.863 & 0.845 & 0.942 & 0.933 & 0.897 & 0.800 & 0.868 & 0.876 \\
\hline
\end{tabular}

A - Supegaon; B - Barshiv; C - Par Gaan; D - Chikhal Gaan; E - Phansad Gaan; F - Savarat Gaan; G - Chakacha Maal; H - Ghunyacha Maal.

Table 5. Changes in species abundance, Shannon index and evenness index of amphibians in total study period.

\begin{tabular}{|l|c|c|c|c|}
\hline Month & June & July & Aug & Sep \\
\hline Species & 21 & 20 & 21 & 15 \\
\hline Individuals & 1167 & 679 & 769 & 645 \\
\hline Shannon diversity index $\left(H^{\prime}\right)$ & 1.144 & 1.158 & 1.116 & 0.921 \\
\hline Evenness index & 0.865 & 0.890 & 0.844 & 0.783 \\
\hline
\end{tabular}

evenness index values in September (Table 5) indicates that the frogs in this region prefer earlier three months of wet period to reproduce and at the end of September their breeding activity drastically declines. We have never observed any calling activity of direct developing frogs like Pseudophilautus cf. amboli, Raorchestes bombayensis and Raorchestes cf. bombayensis as well as Wrinkled Frog, Nyctibatrachus humayuni in September. At broad level this might explain that these species prefer highly humid early and mid wet seasons for reproductive activity.

Habitat features plays an important role in governing species diversity and distribution of amphibians (Becker et al. 2007; Santos-Barrera \& Urbina-Cardona 2011). Alteration and loss of habitat structure by multiple anthropogenic activities and changes in land use have been identified as the most critical factors affecting amphibian survival (Cushman 2006; Gardner et al. 2007; Urbina-Cardona 2008). In our study period we have recorded different habitat features at each study site. Table 1 shows that study areas in PWS were divided into three major types of habitats. Supegaon, Barshiv and Par Gaan bears degraded moist deciduous forest, Chakacha Maal and Ghunyacha Maal plateau showing typical grassland habitat while core areas of sanctuary like Chikhal Gaan, Phansad Gaan and Savarat Gaan showing Memecylon umbellatum dominated evergreen type of forest. Table 1 reveals that areas like Supegaon,
Barshiv and Par Gaan are facing anthropogenic threats like human encroachment in sanctuary areas, extensive grazing, monotypic teak wood plantation, deforestation for firewood, slash and burn for shift cultivation (agriculture purpose) etc. In comparison of some physical and biological habitat parameters (Table 1) the Chikhal Gaan, Phansad Gaan and Savarat Gaan stands unique among other study sites in PWS because of atmospheric temperature of these areas ranges between $26-29{ }^{\circ} \mathrm{C}$ (vs. $28-33{ }^{\circ} \mathrm{C}$ in other areas), Humidity varies between 88-97 \% (vs. 68-86 \%) except Chakacha Maal and Ghunyacha Maal, canopy cover stands between 60-91 $\%$ (vs. 20-60\%), riparian canopy cover varies between 75-91\% (vs. 0-63 \%) and forest floor litter depth stands between $70-210 \mathrm{~mm}$ (vs. 0-70 $\mathrm{mm}$ at other sites). For implementation of systematic conservation efforts in the Western Ghats, biodiversity hotspot prioritization of highly diverse areas is essential (Myers 1990; Myers et al. 2000; Dahanukar \& Padhye 2005; Mittermeier et al. 2005). Analysis of species assemblage similarity (Fig. 3) between study areas reveals Chikhal Gaan and Phansad Gaan forming a single and distinct cluster while other study areas remains separated.

Environmental parameters play an important role in species distribution, therefore understanding relationships between habitat characteristics and species distribution pattern is vital for developing effective conservation strategies for threatened taxa (Boyd et al. 2008). Anthropogenic activities like deforestation, construction of dams, human encroachment, slash and burn techniques for shifting cultivation, road construction and mining. had been known to be possible causes of changes in the habitat structure at landscape level which ultimately affects species diversity and their assemblage (Gururaja et al. 2008). It is already known that habitat destruction due to several anthropogenic activities has had a negative impact on amphibian diversity and distribution in Western Ghats (Krishnamurthy 1996, 


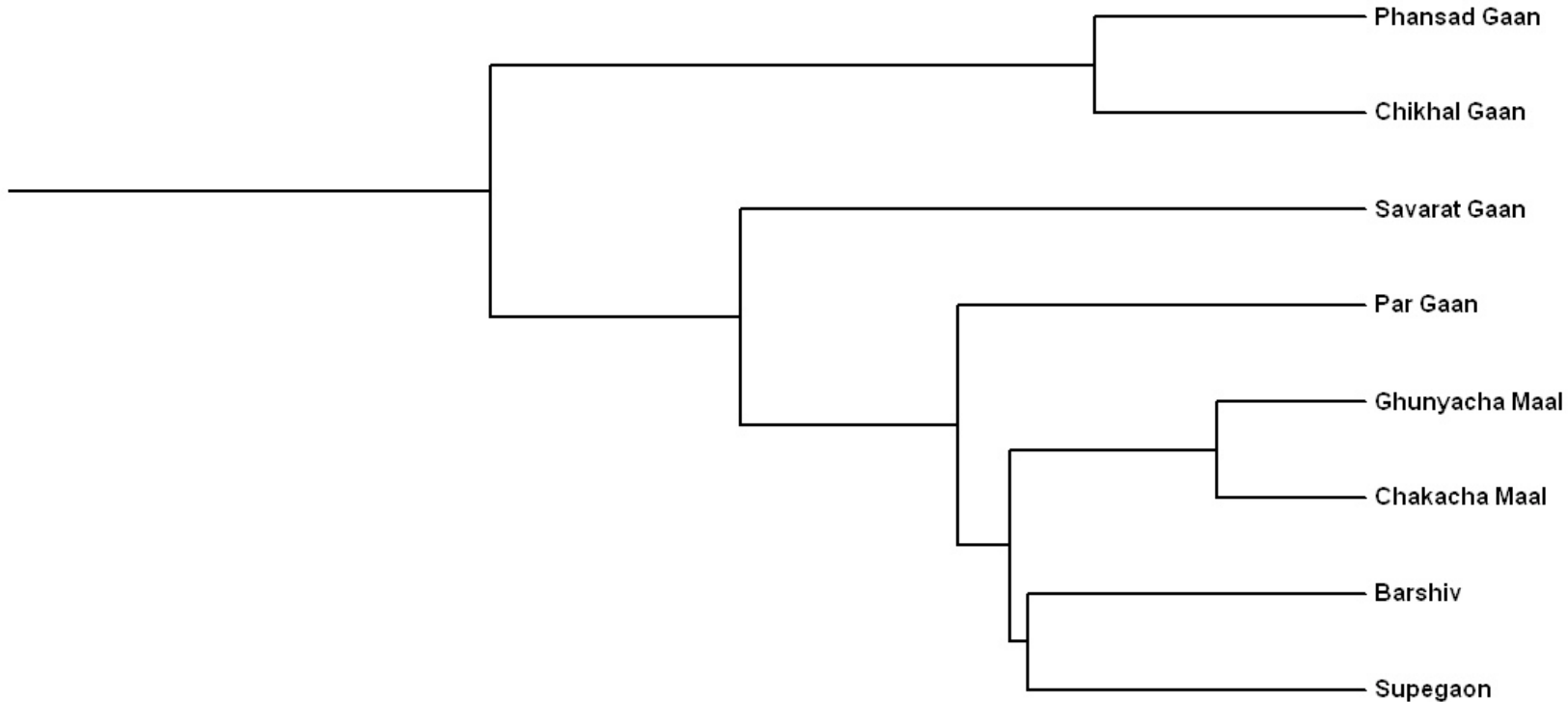

Figure 3. Dendrogram based on transect data analyzed by using Bray-Curtis similarity index, representing species assemblage structure similarity between different study areas of Phansad Wildlife Sanctuary.

2003; Padhye et al. 2002; Dahanukar \& Padhye 2005; Krishnamurthy \& Reddy 2008). Relationship between anuran species, study sites and habitat variables were predicted by using canonical correspondence analysis (CCA). Axis $1 \& 2$ represents species points which are determined by weighted average of axis values of preferred habitat variables and study sites in which they were occurred. CCA analysis extracted different axis scores for species point, study site and for habitat variables. Two best axis scores representing maximum variation in data were selected to draw the ordination plot (Fig. 4). Species points in this triplot formed their unique niche clusters with respect to different study locations and habitat variables. Nyctibatrachus humayuni, Sphaerotheca dobsonii, Indirana beddomii, I. leithii, Polypedates cf. maculatus, Pseudophilautus cf. amboli, Raorchestes bombayensis and R. cf. bombayensis forms a distinct species cluster showing two dimensional unique niche because they share similar type of habitat and prefers more humid evergreen forest patches. Like species points, similar habitat sharing study sites also form their clusters. CCA ordination triplot indicates variables like canopy cover, riparian canopy cover, forest floor litter depth, humidity, temperature, stream persistence and forest type shows high negative correlation with axis 1 . Species and study sites present in ordination with axis 1 have high influence of environmental variables. Variable like altitude found to be positively correlated on axis 2. Species like Duttaphrynus melanostictus, Euphlyctis cyanophlyctis, Fejervarya cf. keralensis, F. syhadrensis, Hoplobatrachus tigerinus, Minervarya sahyadris, Sphaerotheca breviceps, Ramanella mormorata and Uperodon globulosus were found to be present in human disturbed areas like Supegaon, Barshiv and Par Gaan as well as it has been found that they are independent from habitat variable influence.

On a broad scale our current analysis of anuran distribution in PWS with respect to different study localities and habitat parameters (Fig. 4) indicates that anuran species like Nyctibatrachus humayuni, Indirana beddomii, I. leithii, Polypedates cf. maculatus, Pseudophilautus cf. amboli, Raorchestes bombayensis and $R$. cf. bombayensis were restricted to evergreen forest areas like Chikhal Gaan, Phansad Gaan and Savarat Gaan because of their specific habitat requirements like dense canopy coverage, maximum forest floor litter, low atmospheric temperature and high humidity. Whereas species like Duttaphrynus melanostictus, Euphlyctis cyanophlyctis, Fejervarya cf. keralensis, F. syhadrensis, Hoplobatrachus tigerinus, Minervarya sahyadris and Sphaerotheca breviceps showing maximum occurrence in human disturbed areas like Supegaon, Barshiv and Par Gaan having minimum canopy cover, minimum forest floor litter, low humidity and comparatively high 


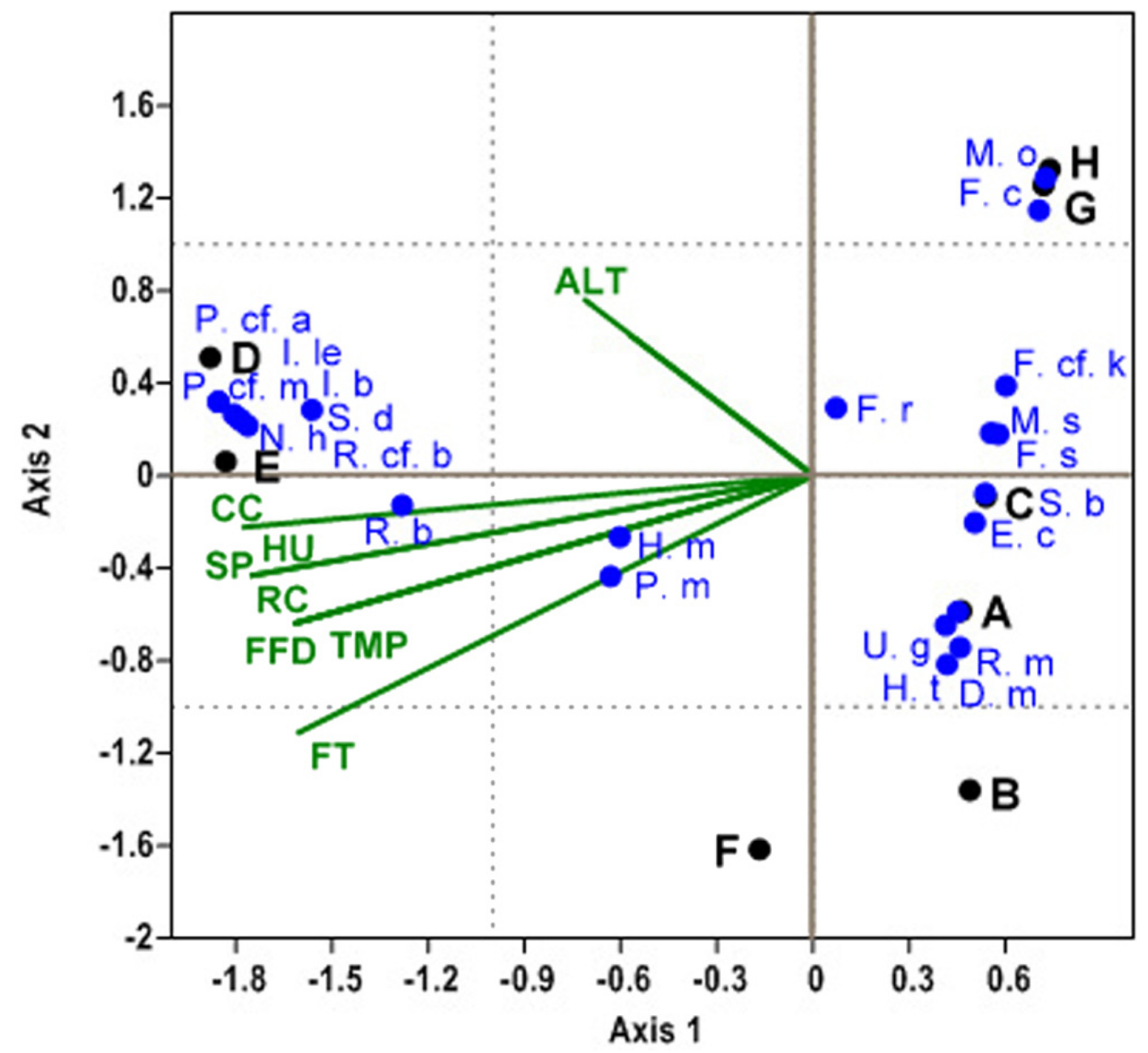

Figure 4. Canonical Correspondence Analysis (CCA) ordination triplot showing anuran species distribution with reference to study sites and habitat variables. Site labels are coded in Figure 1. Habitat variable codes: ALT - altitude; CC - canopy coverage; RC - riparian canopy coverage; HU - humidity; SP - stream persistence; FFD - forest floor litter depth; TMP - temperature; FT - forest type. Species codes: D. m - Duttaphrynus melanostictus; E. c - Euphlyctis cyanophlyctis; F. cf. k - Fejervarya cf. keralensis; F. c - Fejervarya caperata; F. r - Fejervarya rufescens, F. s - Fejervarya syhadrensis, H. t - Hoplobatrachus tigerinus; M. s - Minervarya sahyadris; S. b - Sphaerotheca breviceps; S. d Sphaerotheca dobsonii; M. o - Microhyla ornata; R. m - Ramanella mormorata; U. g - Uperodon globulosus; N. h - Nyctibatrachus humayuni; H. m - Hylarana malabarica; I. b - Indirana beddomii; I. le - Indirana leithii; P. m - Polypedates maculatus; P. cf. m - Polypedates cf. maculatus; P. cf. a - Pseudophilautus cf. amboli; R. b - Raorchestes bombayensis; R. cf. b - Raorchestes cf. bombayensis.

temperature. CCA analysis results indicate that maximum conservation priority should be given to regions having high influence of habitat variables. Priority regions for long term anuran species and habitat monitoring in PWS should be Chikhal Gaan, Phansad Gaan and Savarat Gaan.

In this study we have recorded the occurrence of Minervarya sahyadris (Image 13) from PWS. Identification characteristics like rictal gland present just behind the mouth commissure, white horizontal band along the upper lip, smaller snout to vent length, pointed snout, fold from eye to shoulder, tympanum dark brown in color with inferior border white, digit tips rounded, very less developed webbing and reddish-brown mid dorsum color were observed among specimens recorded from PWS. This endemic species was earlier recorded from Calicut in Kerala and Gundia, Jog, Dandeli, Castle Rock in Karnataka (Dubois et al. 2001; Gururaja 2012). Occurrence of $M$. sahyadris in PWS shows the northward range extension of this species in Western Ghats. Another endemic dicroglossid Fejervarya caperata (Image 14) was also recorded during study. Characters like pointed snout, 20-35 mm snout to vent length, dilations absent on figure or toe tips, presence of fejervaryan line on the sides of abdomen, three distinct cross bars on thigh and four distinct longitudinal ridges on the back were 

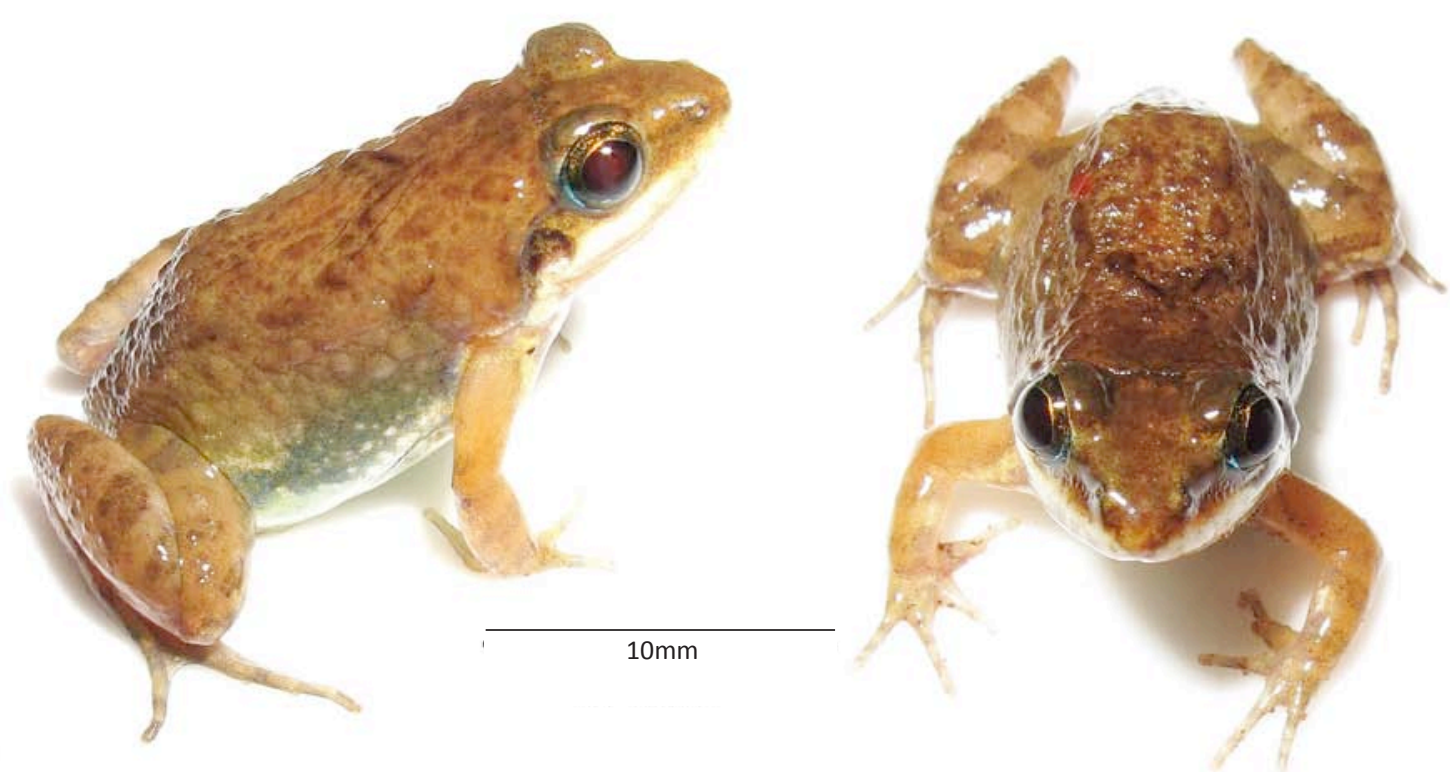

Image 13. Minervarya sahyadris recorded from Phansad Wildlife Sanctuary shows details of its body with (a) lateral and (b) anterior dorsal view.
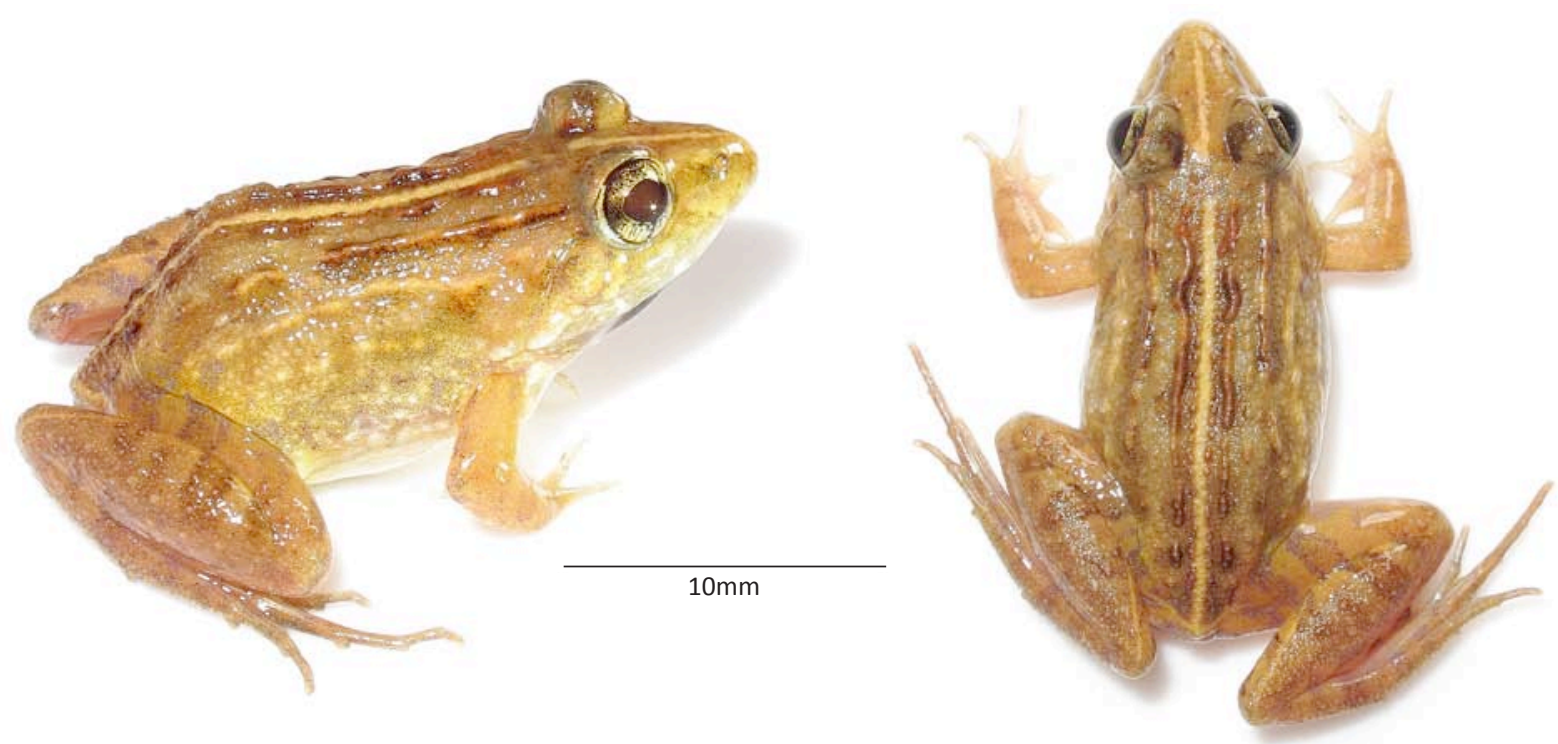

Image 14. Fejervarya caperata sampled during study shows (a) lateral and (b) dorsal portions of its body.

observed among $F$. caperata specimens sampled during the study. Earlier records of this species were found from Karnoor, Bajpe, Shimoga, Jog, and Dandeli in Karnataka parts of Western Ghats (Kuramoto et al. 2007; Gururaja \& Ramachandra 2012; Gururaja 2012). Record of this species in PWS also marks its range extension further north in Western Ghats.

We have reported some frog species from study area having ambiguous taxonomic status. Biju \& Bossuyt (2009) described Pseudophilautus amboli from
Amboli, Sindhudurg District of Maharashtra. Our PWS collections of Pseudophilautus cf. amboli differs from Pseudophilautus amboli (Biju \& Bossuyt 2009) sensu stricto in the ratio of head width vs. head length as well in the proportion of third finger disc diameter vs. finger width. In consideration of such ambiguities further study is required to obtain presence of this species in PWS. Some population of Raorchestes bombayensis from our collection differs from original description of Annandale (1919) and latest reviewed description given 
by Biju \& Bossuyt (2009). Raorchestes bombayensis (Annandale 1919; Biju \& Bossuyt 2009) sensu stricto differs from our PWS collection in having head width greater than head length (vs. head width almost equal to head length) and smaller eye length. Occurrence of Fejervarya cf. keralensis also needs further confirmation as it lacks spinular projections on thigh region. We have also collected some specimens of tree frogs Polypedates from our study area. Although some of our specimens matches very closely with description given by Daniel \& Sekar (1989) for Polypedates maculatus, but they also differ in having comparatively smaller snout to vent length, tympanum almost equal to eye diameter and distinct striped banding pattern on dorsum.

Global threat status analysis of anurans recorded in this study (Table 3) shows that about seven species (32\%) are threatened (Vulnerable (VU), Endangered (EN) or Critically Endangered (CR)). Fourteen species come under Least Concern (LC) category while one is Data Deficient (DD). Occurrence of CR species like Pseudophilautus of. amboli as well as some EN and VU species like Minervarya sahyadris, Nyctibatrachus humayuni, Indirana leithii and Raorchestes bombayensis is important from conservation point of view.

In conclusion occurrence of around $50 \%$ endemic anuran species of which $32 \%$ are globally threatened marks PWS as key site for amphibian diversity in Western Ghats biodiversity hotspot. Implementation of strong conservation measures and policies are needed in PWS for amphibian conservation. This study would be beneficial in this context.

\section{REFERENCES}

Annandale, N. (1919). The fauna of certain small streams in the Bombay presidency. Records of Indian Museum 16: 109-161.

Becker, C.G., C.R. Fonseca, C.F.B. Haddad, R.F. Batista \& P.I. Prado (2007). Habitat split and the global declines of amphibians. Science 318: 1775-1777.

Bhatta G. \& P. Prashanth (2004). Gegeneophis nadakarnii - a caecilian (Amphibia: Gymnophiona: Caeciliidae) from Bondla wild life sanctuary, Western Ghats. Current Science 87(3): 388-392.

Bhatta, G., K.P. Dinesh, P. Prashanth \& N.U. Kulkarni (2007). A new species of Gegeneophis Peters (Amphibia: Gymnophiona: Caeciliidae) from Goa, India. Zootaxa 1409: 51-59.

Biju S. D., I.V. Bocxlaer, S. Mahony, K.P. Dinesh, C. Radhakrishnan, A Zachariah, V. Giri \& F. Bossuyt (2011). A taxonomic review of the Night Frog genus Nyctibatrachus Boulenger, 1882 in the Western Ghats, India (Anura: Nyctibatrachidae) with description of twelve new species. Zootaxa 3029: 1-96.

Biju, S.D. \& F. Bossuyt (2009). Systematics and phylogeny of Philautus Gistel, 1848 (Anura, Rhacophoridae) in the Western Ghats of India, with descriptions of 12 new species. Zoological Journal of the Linnean Society 155: 374-444.

Bossuyt, F. \& A. Dubois (2001). A review of the frog genus Philautus Gistel, 1848 (Amphibia, Anura, Ranidae, Rhacophorinae). Zeylanica
6: 1-112

Boulenger, G.A. (1890). Fauna of British India Including Ceylon and Burma, Reptilia and Batrachia. Taylor and Francis, London.

Boyd, C., T.M. Brooks, S.H.M. Butchart, G.J. Edgar, G.A.B. da Franseca, F. Hawkins, M. Hoffmann, W. Sechrest, S.N. Stuart \& P.P. van Dijk (2008). Spatial scale and the conservation of threatened species. Conservation Letters 1: 37-43.

Colwell, R.K. (2006). EstimateS: Statistical estimation of species richness and shared species from samples. Version 8.0.0. User's Guide and application published at: http://purl.oclc.org/estimates.

Cushman, S.A. (2006). Effects of habitat loss and fragmentation on amphibians: A review and prospectus. Biological conservation 128: 231-240.

Dahanukar, N. \& A. Padhye (2005). Amphibian diversity and distribution in Tamhini, northern Western Ghats, India. Current science 88(9): 1496-1501.

Daniel, J.C. \& A.G. Sekar (1989). Field guide to the amphibians of western India, Part 4. Journal of the Bombay Natural History Society 86: 194-203.

Daniel, J.C. (1963 a). Field guide to the amphibians of western India. Part 1. Journal of the Bombay Natural History Society 60: 415-438.

Daniel, J.C. (1963 b). Field guide to the amphibians of western India. Part 2. Journal of the Bombay Natural History Society 60: 690-702.

Daniel, J.C. (1974). Amphibia. In: Kunte, B.G. (ed.). Gazetteer of India, Maharashtra-State Gazetteers, General series: Fauna. Government of Maharashtra publication.

Daniel, J.C. (1975). Field guide to the amphibians of western IndiaPart 3. Journal of the Bombay Natural History Society 72: 506-522.

Daniels, R.J.R. (1991). The problem of conserving amphibians in the Western Ghats, India. Current science 60: 630-632.

Daniels, R.J.R. (1992). Geographical range and ecology of the Verrucose Frog Rana keralensis (Dubois). Journal of the Bombay Natural History Society 89: 199-203.

Dubois, A., A. Ohler \& S.D. Biju (2001). A new genus and species of Ranidae (Amphibia, Anura) from south-western India. Alytes 19(24): 53-79.

Frost, R.D. (2011). Amphibian Species of the World: an Online Reference. Version 5.5 (22 October 2011). Electronic Database accessible at http://research.amnh.org/vz/herpetology/amphibia/ American Museum of Natural History, New York, USA.

Gardner, T.A., J. Barlow \& C.A. Peres (2007). Paradox, presumption and pitfalls in conservation biology: The importance of habitat change for amphibians and reptiles. Biological Conservation 138: 166-179.

Ghate, H.V. \& A.D. Padhye (1996). Impact of urbanization on amphibians of Pune. Zoos' Print XI: 14-16.

Giri, V., D.J. Gower \& M. Wilkinson (2004). A new species of Indotyphlus Taylor (Amphibia: Gymnophiona: Caeciliidae) from the Western Ghats, India. Zootaxa 739: 1-19.

Gokhale, V.V. \& R.A. Velankar (1996). Documenting Peoples' Knowledge about Biodiversity at Supegaon Village. RANWA Technical Report 6.

Gunawardene, N.R., A.E.D. Daniels, I.A.U.N. Gunatilleke, C.V.S. Gunatilleke, P.V. Karunakaran, K.G. Nayak, S. Prasad, P. Puyravaud, B.R. Ramesh, K.A. Subramanian \& G. Vasanthy (2007). A brief overview of the Western Ghats - Sri Lanka biodiversity hotspot. Current Science 11: 1567-1572.

Gururaja, K.V. (2012). Pictorial Guide to Frogs and Toads of the Western Ghats. Gubbi Labs, India, xviii+153pp.

Gururaja, K.V., S. Ali \& T.V. Ramachandra (2008). Influence of Land-use Changes in River Basins on Diversity and Distribution of Amphibians. Environmental Education for Ecosystem Conservation. Capital publishing company, New Delhi.

Gururaja, K.V. \& T.V. Ramachandra (2012). Anuran Diversity and Distribution in Dandeli Anshi Tiger Reserve. Sahyadri Conservation Series: 8 ENVIS Technical Report 37.

Hammer, Ø., D.A.T. Harper \& P.D. Ryan (2001). PAST: Paleontological Statistics software package for education and data analysis. Palaeontologia Electronica 4(1):9.

Hijmans, R.J., L. Guarino, E. Rojas (2002). DIVA-GIS a geographic 
information system for the analysis of biodiversity data. Manual, International Potato Center, Lima, Peru.

IUCN (2011). Guidelines for appropriate uses of IUCN Red List Data. Incorporating the Guidelines for Reporting on Proportion Threatened and the Guidelines on Scientific Collecting of Threatened Species. Version 2. Adopted by the IUCN Red List Committee and IUCN SSC Steering Committee. Downloadable from: http://intranet. iucn.org/webfiles/doc/SpeciesProg/RL_Guidelines_Data_Use.pdf Downloaded on 10 September 2011.

IUCN (2011). IUCN Red List of Threatened Species. Version 2011.1 <www.iucnredlist.org>. Downloaded on 09 August 2011.

Krishnamurthy, S.V. (1996). Habitat features of amphibians in Sringeri, Western Ghats. Zoos' Print 8: 2-6.

Krishnamurthy, S.V. (2003). Amphibian diversity and consequences of habitat dissimulation on their distribution in central Western Ghats of Karnataka. SERC Research Highlights, Department of Science and Technology, India, 9-38.

Krishnamurthy, S.V. \& A.H.M. Reddy (2008). An estimate of local population of Nyctibatrachus aliciae at two habitat gradients of forest in Western Ghats. Acta Herpetologica 3: 51-55.

Kumbar, S.M. \& S.S. Patil (2010). Checklist and habitat of anuran species in the Sangli District, Maharashtra. Frog leg 14: 21-24.

Kuramoto, M., S.H. Joshy, A. Kurabayashi, M. Sumida (2007). The genus Fejervarya (Anura: Ranidae) in central Western Ghats, India with descriptions of four new cryptic species. Current Herpetology 26(2): 81-105.

Magurran, A.E. (1988). Ecological Diversity and its Measurement. Chapman and Hall, London, 192pp.

Mittermeier, R.A., P.R. Gil, M. Hoffman, J. Pilgrim, T. Brooks, C.G. Mittermeier, J. Lamoreux \& G.A.B. da Fonseca (2005). Hotspots Revisited: Earth's Biologically Richest and most Endangered Terrestrial Ecoregions. Cemex, Mexico, 392pp.

Myers, N. (1990). The biodiversity challenge: expanded hotspots analysis. The Environmentalist 10: 243-256.

Myers, N., R.A. Mittermeier, C.G. Mittermeier, G.A.B. Da Fonseka \& J. Kents (2000). Biodiversity hotspots for conservation priorities.
Nature 403: 853-858

Nair, A., O. Daniel, S.V. Gopalan, S. George, K.S. Kumar, J. Merilä \& A.G.F. Teacher (2011). Infectious disease screening of Indirana frogs from the Western Ghats biodiversity hotspot. Herpetological Review 42: 554-557.

Padhye, A.D., M. Mahabaleshwarkar \& H.V. Ghate (2002). An overview of amphibia fauna of Pune district with special reference to their status in and around Pune city. Zoos' Print Journal 17(4): 757-763.

Padhye, A.D. \& H.V. Ghate (2002). An overview of amphibian fauna of Maharashtra state. Zoos' Print Journal 17(3): 735-740.

Pande, P. \& N. Pathak (2005). National Parks and Sanctuaries in Maharashtra. Bombay Natural History Society, Volume II, 403413pp.

Ravichandran, M.S. \& R.S. Pillai (1990). Amphibia of Maharashtra with description of a new species of Torrent Toad, Ansonia. Records of the Zoological Survey of India 86: 505-513.

Rodgers, W.A. \& H.S. Panwar (1988). Planning A Wildlife Protected Area Network in India. Volume 2. Wildlife Institute of India, Dehra Dun.

Santos-Barrera, G. \& J.N. Urbina-Cardona (2011). The role of the matrix-edge dynamics of amphibian conservation in tropical montane fragmented landscapes. Revista Mexicana de Biodiversidad 82: 679-687.

Sekar, A.G. (1999). Four new records and checklist of amphibians from Maharashtra. Journal of the Bombay Natural History Society 96(1): 152-157.

Seshadri, K.S., K.V. Gururaja \& N.A. Aravind (2012). A new species of Raorchestes (Amphibia: Anura: Rhacophoridae) from mid-elevation evergreen forests of the southern Western Ghats, India. Zootaxa 3410: 19-34.

Urbina-Cardona, J.N. (2008). Conservation of Neotropical herpetofauna: research trends and challenges. Tropical Conservation Science 1: 359-375.

Yazdani, G.M. \& A. Mahabal (1976). Amphibians of Poona. Newsletter of the Zoological Survey of India 2: 138-139. 